\title{
Internet connected wireless combustible gas monitoring system for apartment buildings
}

\author{
Denis Spirjakin \\ LLC "Smartsense", \\ Moscow, Russia \\ Email: denis.spirjakin@gmail.com
}

\author{
Alexander M. Baranov \\ Moscow Aviation Institute \\ (National Research University), \\ Moscow, Russia
}

\begin{abstract}
Despite the modern gas equipment, combustible gas leakage related emergency situations still take place and lead to building demolitions and human losses. Leak integrity failures because of anthropogenic and natural factors make impossible to prevent such emergency in other ways except providing continuous monitoring of combustible gas concentration and notification for people and special services. In this work, the design results of the Internet connected wireless sensor network for combustible gas concentration monitoring in apartment buildings is presented. The system consists of wireless autonomous gas sensors, actuators, routers and a gateway and it's connected to a web service where it posts its data and gets events to react them in WSN.
\end{abstract}

\section{INTRODUCTION}

E VEN though modern gas equipment have flame failure control systems, combustible gas leakage related emergency situations still take place quite often. In the worst case these situations can lead to building demolitions and human losses. The main reason for those emergency situations is leak integrity failures which happen because of human factor, pipes and pipe joints quality and corrosion, etc [1]. To prevent such situations it's necessary to provide continuous monitoring of combustible gas concentration and timely notification for people and special services.

Wireless sensor networks becomes more and more popular and a lot of them were designed recently [2] - [5]. These networks consist of small nodes and are equiped with transceivers, microprocessors and sensors and can be used in different areas of life (safety, military, home automation, etc.).

Wireless sensor networks have certain advantages to perform the monitoring. Wireless autonomous devices don't need wiring and can be easily distributed inside apartment building and the network can freely be extended when needed. Established wireless network can provide additional services in home automation and others.

Wireless sensor networks are widely used in gas concentrations monitoring [6] - [9]. The main problem for wireless autonomous sensor devices is high energy consumption of combustible gas sensors [10]. This problem can be solved using special measuring algorithms [11].

According to safety standards [12] notification system of a gas detection device should include audio signal with specified sound level and visual signals to indicate power on and alarm states. This mandatory notification system can be extended by sending alarm notification to an operator console and cell phones over cellular networks using both short message service and Internet connection.

In the case of Internet connection, data is posted to a web service and any device connected to the Internet can get access to it. The conception of devices, connected together and with their users, is known as Internet of Things [13]. This way makes possible to extend WSN automation and notification functions, for example, providing additional notifications from utility companies about maintenance period, or remote control functions to any device connected to WSN.

Therefore, the most promising approach to monitor methane leakages in apartment buildings is using electronic systems with wireless data transmission. There are several most popular technologies for wireless communication: ZigBee, Bluetooth, GSM/GPRS and Wi-Fi [14], [15]. The most important problems of the majority of such systems are the

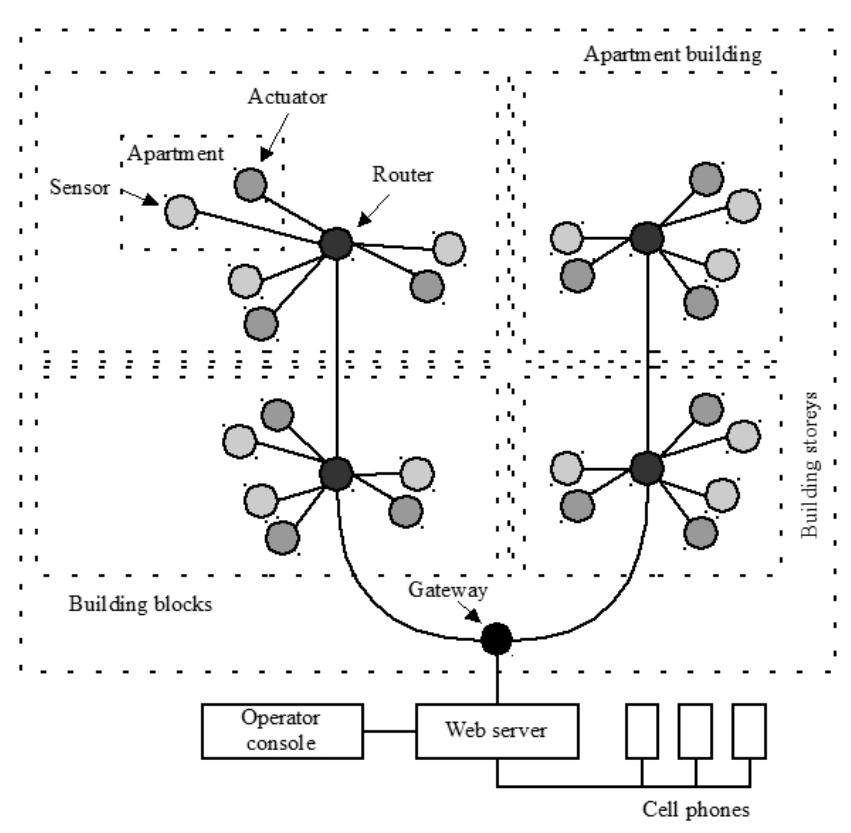

Fig 1. System diagram 


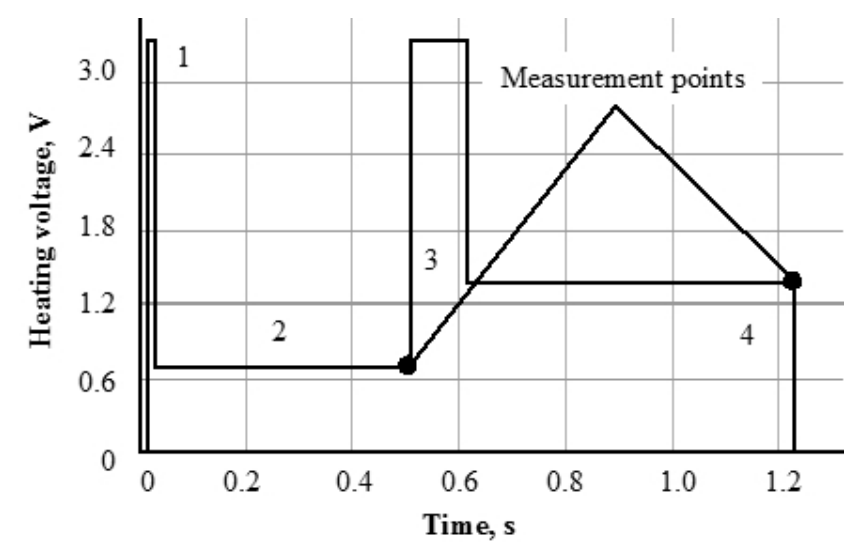

Fig 2. Heating voltage

limited access to data and short autonomous lifetime of the gas sensors.

In this work, the design results of the Internet connected wireless sensor network for combustible gas concentration monitoring in apartment buildings is presented. The system consists of wireless autonomous gas sensors, actuators, routers and a gateway and it's connected to a web service where it posts its data and gets events to react them in WSN. The system is used to equip a trial apartment building in Moscow region.

The paper is organized as follows. At first we overview the system in Section II. In Section III we describe the nodes design. Section IV is dedicated to the network organization. Internet connectivity is discussed in Section V. Finally, we provide concluding remarks in Section VI.

\section{System Overview}

The system consists of the following devices: gas sensors, actuators, routers and a gateway. The system block diagram is presented in Fig. 1. Wireless communication between devices complies IEEE 802.15.4/ZigBee standards and uses unlicensed $2.4 \mathrm{GHz}$ ISM band. Internet connection is performed on the gateway using cellular networks.

There is only one gateway for a building. It creates the network and acts like a network coordinator. Except that, it's also a sink device and all sensors send data to it.

Since distances in the building are relatively long, routers are used to transfer data from sensors to the gateway. They are deployed on building storeys, one per a storey in every building block.

The sensors and actuators are deployed in every apartment of the building. Sensors are placed in the kitchen. Actuators are equipped with gas valves and located at the gas pipe entrance point. Except valves, actuators can be connected to a power grid to control power of electrical devices.

The data sent to the gateway is transferred to a web service on the Internet and stored in a database. The service uses REST API to send and retrieve data for machines and a web interface for human clients.
Users of the system are presented by apartment residents and special service operators. All users have their own accounts with their own restrictions. For example, apartment residents have access only to devices in their apartments, the special services have access to all gas sensors and a valve actuator at the gas pipe entrance point of the building.

\section{Nodes Design}

\section{A. Sensor}

To perform combustible gases concentration measurements the node uses ATxmega16E5 microcontroller and the commercial catalytic gas sensor. The sensor is manufactured by NTC IGD (Russia) and its power consumption is $110 \mathrm{~mW}$ in continuous measurement mode. Since, the catalytic gas sensor is very power hungry, to decrease its power consumption the measurements are performed in periodical mode and the special measuring algorithm is used.

The wireless communication is performed using Telegesis ETRX3 module. It provides the IEEE 802.15.4/ZigBee standards compatible protocol and has UART control interface with AT-style commands set.

The node is battery powered. It uses AA size lithium battery with $3.6 \mathrm{~V}$ nominal voltage and $2600 \mathrm{mAh}$ capacity. To provide maximum efficiency in voltage conversion it is performed by a TPS63060 DC-DC converter. The device generates output voltage of $3.2 \mathrm{~V}$ from $2.5 \mathrm{~V}$ to $12 \mathrm{~V}$ on its input with load regulation of $0.5 \%$ with power save mode disabled.

To maintain the low power consumption the measurements are performed periodically and special multistage pulse algorithm with PWM heating is used. This method was offered and discussed in [11].

The heating profile for the pulse is shown in Fig. 2. In the first and second stages of the profile the sensor is heated up to about $200 \mathrm{C}$ where catalytic reaction is kinetic-controlled and there is no additional heating of sensing element from target gas combustion. During the third and fourth stages the sensor heating is continued and its temperature rises up to

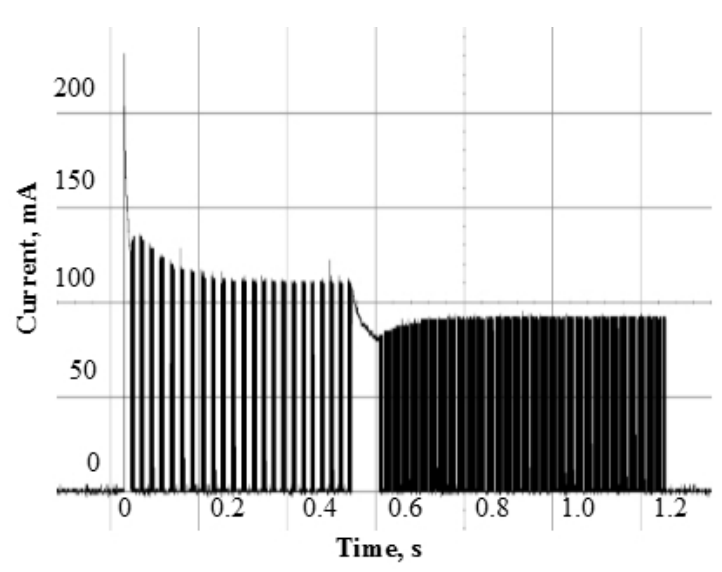

Fig 3. Multistage pulse measurements current consumption 
the catalysis external diffusion region (about 450C). The heating voltage values for the stages are $3.2 \mathrm{~V}, 0.7 \mathrm{~V}, 3.2 \mathrm{~V}$ and $1.4 \mathrm{~V}$ respectively. First and third of them are forcing the heating process, second and fourth ensure the target temperature stabilization.

The measuring circuit is controlled by the microcontroller and includes two MOSFET keys to connect the sensor to the heating voltage and to the reference resistor. To heat the sensor up the microcontroller forms PWM signal with $10 \mathrm{kHz}$ frequency and appropriate duty cycle corresponding to specific heating voltage. Measurements are performed between the heating pulses. The reference resistor - sensor divider is connected to the microcontroller ADC, where the steady component is subtracted from the signal and the result is amplified with buit-in amplifier and converted. The measurement result is the difference between sensor voltages at two different temperatures (measurement points in the heating profile diagram).

The sensor sensitivity in this case is $1.8 \mathrm{mV} / \mathrm{vol} \%$. The node operates as a two-threshold device. Threshold values are 0.5 and $1 \mathrm{vol} \%$. When the sensor crosses the threshold, the node enables alarm signals and sends the data to the gateway.

To maintain the low power consumption the sensor node performs measurements periodically once in 5 minutes. Between measurements the microcontroller disables power of all components and goes to the power save mode.

The current consumption of the measuring pulse is presenting in Fig. 3. The duty cycles during the second and fourth stages are 22 and 44 percents. Therefore, the average current consumption value for the pulse is $37 \mathrm{~mA}$ during 1.2 seconds.

To maintain online status the node sends the data every working cycle. The average current consumption in this state is $38 \mathrm{~mA}$ during 0.35 seconds.

The average current consumption for the full cycle is $0.19 \mathrm{~mA}$. With the battery capacity of $2.6 \mathrm{Ah}$ the node autonomous lifetime is equal to 570 days.

\section{B. Actuator}

The main function of an actuator is to control a gas valve at a gas pipe entrance point. It also can be connected to a power grid to control power of electrical devices.

The device is line powered. The power supply generates voltages to supply a digital control circuit, electromagnetic switches and external outputs.

The digital control circuit consists of a STM32F102C6 microcontroller to process commands and a Telegesis ETRX3 module for the WSN communication. The supply voltage of the circuit is $3.3 \mathrm{~V}$. To control electromagnetic switches with $12 \mathrm{~V}$ switching voltage the MOSFET keys are used.

The actuator provides four outputs to control external circuits. Two of them are connected to power sources with $12 \mathrm{~V} / 1 \mathrm{~A}$ and regulated voltage. These outputs are originally intended to control gas valves of different types, but can be also used to drive another electronic devices. Another two outputs can be connected to a power grid and commutate it.

\section{Gateway and routers}

Even though the transmission area of the communication module is up to 350 meters without obstacles, walls and floor decks significantly reduce the distance. Coexistence with other networks, like Wi-Fi, can decrease it even more. Routers provide a steady way to transfer data between nodes.

Both routers and gateways are line powered and consist of a power supply, a STM32F102C6 microcontroller and a Telegesis ETRX3 module. The gateways have additional Telit GL865-QUAD GSM/GPRS communication module to connect to the Internet.

\section{Network Organization}

The network uses a tree topology. The main device of the network is a gateway. The gateway always creates the network and announce itself as a sink device. Other devices request the gateway address during connection process.

The gateway and routers are full functional devices. That means they are always ready to receive data, can be a parent node for other nodes and can perform routing. All other devices of the network are end devices and can't have child nodes.

All sensors send data to the gateway. The data is sent only when sensor states are changed. But every work cycle all devices should announce that they are alive and connected to the network. If a node haven't sent an alive message in predefined timeout, it's considered to be offline and the system generates alarm notification.

\section{InTERnEt ConNeCtivity}

The gateway sends data to a web service on the Internet where it's stored in a database. To send the data HTTP protocol is used. To be safe the transmission is performed over TLS protocol. The protocols realization is provided by GSM/GPRS communication module.

The web service uses REST API to send and retrieve data for machines and a web interface for human clients. It is based on Apache HTTP Server and uses CMS Drupal and MySQL database server.

CMS Drupal 8 is a flexible content management software for websites and web applications. It's modular and can easily be extended. Drupal has built-in RESTful API with both JSON and XML support to integrate it with external services.

All devices are represented in the service by separate content type - Device Object. This content type have the following fields to represent its parameters: Groups, Network class, Device address, Subdevice address, Status and Value. Groups field contains information about groups associated with a device. Network class is used to create separate address space for networks with different standards and virtual devices. Device address field represents the network device 
address (e.g., IEEE 802.15.4 MAC address). Subdevice address is used to distinguish different parts of a device with the same network address. Status field represents the network status of the device. And Value field contains an integer value.

Devices are connected to each other with event system. Events are also represented by a special content type - Event Object. Event Object contains the following fields: Source device, Target device, Status, Operation, Parameter, Value and Notification. Source device contains the information about a device which created the event. Target device points to a device which should process the event. Status field contains the event status. It's equal to 1 if it's a pending event, and to 0 if there is no need to process it. Operation and Parameter fields define the way the event is processed. Operation can contain one of the following actions: more, less, equal and changed. This actions are applied to the source device value. The comparison is performed with $\mathrm{Pa}$ rameter field. When Operation is "changed", the Parameter is ignored. Value field contains the data to assign to the target device. And Notification field passes the notification message to the event handler.

The event system is managed with Drupal module. All devices of the network have their own Drupal nodes. When a device node is changed, the event system looks for associated events. If it finds any, the status of that event is changed to 1 (pending event).

Gateways regularly lookup the event list for pending events. When such event exists, a gateway requests all information about it and process it. After processing the gateway modify the target device and sets the event status value to 0 .

Device nodes are grouped to reflect the apartment building and the apartment it belongs. Since all devices and events are nodes, they are affected by built-in access control system of Drupal. All users have their own restrictions based on device groups. But gateways use a separate account to have write access to all records.

\section{Conclusion}

In this paper, a wireless system for combustible gas leakage monitoring in apartment buildings is presented. The system consists of autonomous gas sensors, actuators, routers and a gateway and is connected to the Internet. The architecture of the system allows to adjust the number of nodes depending on the number of apartments. The gas concentration data is stored in the web service database on the Internet and can be easily accessed from any mobile device (laptop, tablet PC, smartphone, etc.).

The system response time is determined by the duty cycle of methane concentration measurements and can vary in different tasks. The peculiarity of the system is in relocation of network organization, event management and data storage functions to a web service in strong interaction with WSN.
To increase the autonomous lifetime we are going to use alternative energy sources for components of the wireless system in the nearest future [16].

\section{REFERENCES}

[1] Huang Z., Li J. Assessment of fire risk of gas pipeline leakage in cities and towns //Procedia Engineering. - 2012. - T. 45. - C. 77-82. https://doi.org/10.1016/j.proeng.2012.08.124

[2] Kapitulík J., Miček J., Jurečka M., Hodoň M. (2014). Wireless sensor network-value added subsystem of ITS communication platform. In Computer Science and Information Systems (FedCSIS), 2014 Federated Conference on (pp. 1017-1023). IEEE. https://doi.org/10.15439/2014F370

[3] Pei Zhou, Gongsheng Huang, Linfeng Zhang, Kim-Fung Tsang, "Wireless sensor network based monitoring system for a large-scale indoor space: data process and supply air allocation optimization", Energy and Buildings, Volume 103, 15 September 2015, Pages 365374. http://dx.doi.org/10.1016/j.enbuild.2015.06.042

[4] Ferdoush Sheikh, and Xinrong Li. "Wireless sensor network system design using Raspberry Pi and Arduino for environmental monitoring applications." Procedia Computer Science 34 (2014): 103-110. https://doi.org/10.1016/j.procs.2014.07.059

[5] Gutiérrez J., Villa-Medina, J. F. Nieto-Garibay, A., Porta-Gándara M. Á. (2014). Automated irrigation system using a wireless sensor network and GPRS module. IEEE transactions on instrumentation and measurement, 63(1), 166-176. https://doi.org/10.1109/TIM.2013.2276487

[6] A. Somov, A. Baranov, D. Spirjakin, A. Spirjakin, V. Sleptsov, R. Passerone, "Deployment and evaluation of a wireless sensor network for methane leak detection", Sensors and Actuators A: Physiscal 202 (2013) 217-225. http://dx.doi.org/10.1016/j.sna.2012.11.047

[7] Olešnaníková Veronika, Peter Ševčík, and Peter Šarafín. "Monitoring of CO 2 amount in closed objects via WSN." Computer Science and Information Systems (FedCSIS), 2015 Federated Conference on. IEEE, 2015.

[8] Jeličić V., Magno M., Paci G., Brunelli D., Benini L. (2011, June). Design, characterization and management of a wireless sensor network for smart gas monitoring. In Advances in Sensors and Interfaces (IWASI), 2011 4th IEEE International Workshop on (pp. 115-120). IEEE. https://doi.org/10.1109/IWASI.2011.6004699

[9] Yang J., Zhou J., Lv Z., Wei W., Song H. (2015). A real-time monitoring system of industry carbon monoxide based on wireless sensor networks. Sensors, 15(11), 29535-29546. https://dx.doi.org/10.3390\%2Fs 151129535

[10] Alexander Baranov, Denis Spirjakin, Saba Akbari, Andrey Somov, "Optimization of power consumption for gas sensor nodes: A survey." Sensors and Actuators A 233 (2015) 279-289. http://dx.doi.org/10.1016/j.sna.2015.07.016

[11] Spirjakin D., Baranov A. M., Somov A., Sleptsov V. "Investigation of Heating Profiles and Optimization of Power Consumption of Gas Sensors for Wireless Sensor Networks". Sensors and Actuators A: Physical 247 (2016) 247-253. http://dx.doi.org/10.1016/j.sna.2016.05.049

[12] Electrical Apparatus for the Detection of Combustible Gases in DomesticPremises. Test methods and performance requirements, EN 50194-1:2009

[13] Miorandi D., Sicari S., De Pellegrini F., Chlamtac I.: "Internet of Things: Vision, Applications and Research Challenges". J. Ad Hoc Networks $10, \quad 1497-1516 \quad$ (2012). http://dx.doi.org/10.1016/j.adhoc.2012.02.016

[14] Abraham S., Li X. A cost-effective wireless sensor network system for indoor air quality monitoring applications//Procedia Computer Science. -34 (2014). -P. 165-171.

[15] Zheng, Z. B. Design of distributed indoor air quality remote monitoring network//Advanced Materials Research. -850-851 (2014). -P. 500-503.

[16] Baranov A. M., Spirjakin D., Akbari S. Somov A., Passerone R. "POCO: 'Perpetual' operation of CO wireless sensor node with hybrid power supply"// Sensors and Actuators A: Physical. 238 (2016) 112121 .https://doi.org/10.1016/j.sna.2015.12.004 